\title{
A Two-Stage Registration Method for Dental Volumetric Data and Mesh Data
}

\author{
Shuai Yang, School of Computer Science and Technology, Tiangong University, Tianjin, China \\ (iD) https://orcid.org/0000-0002-8279-2499 \\ Wenjing Shi, School of Computer Science and Technology, Tiangong University, Tianjin, China \\ Yongzhen Ke, School of Computer Science and Technology, Tiangong University, Tianjin, China \\ Yongjiang Xue, School of Computer Science and Technology, Tiangong University, Tianjin, China
}

\begin{abstract}
Dental computed tomography (CT) images and optical surface scan data are widely used in dental computer-aided design systems. Registration is essential if they are used in software systems. Existing automatic registration methods are either time-consuming or rough, and interactive registration methods are experience-dependent and tedious because of a great deal of purely manual interactions. For overcoming these disadvantages, a two-stage registration method is proposed. In the rough registration stage, the rough translation and rotation matrices are obtained by applying unit quaternion-based method on the points interactively selected from the two types of data. In the precise registration stage, the stridden sampling is used to reduce computational complexity and the proposed registration algorithm with scale transformation is used for precise registration. The proposed method offers a good trade-off between precision and time cost. The experimental results demonstrate that the proposed method provides faster convergence and smaller registration errors than existing methods.
\end{abstract}

\section{KEYWORDS}

Data Sampling, Dental Computer-Aided Design System, Mesh Data, Two-Stage Registration, Volumetric Data

\section{INTRODUCTION}

Under emerging computer-aided design (CAD), as technology computed tomography (CT) and 3D scanning mature, digital design has gained popularity in multi-tasking, including orthodontic planning and simulation, dental implants, and jaw surgery (Hämmerle et al., 2015; Van Noort, 2012; Wu \& Zhang, 2016; Zhang et al., 2017; Zhao et al., 2017).

The dental volumetric data may be typically obtained by cone-beam computed tomography (CBCT). The volumetric data contain complete dental tissues, such as alveolar bone, tooth crown, tooth root, and other soft tissue. Yet, for various reasons, the volumetric data of CBCT scans have many metal artifacts and defects (Gao \& Chae, 2010; Pauwels et al., 2013), thereby making CBCT images insufficient to highlight the detailed geometries. The dental mesh data, generally triangular mesh, are obtained by 3D optical scanning. While such data are more accurate than dental volumetric data, they only include the tooth crowns and parts of gums; hence, in many dental applications, both the dental volumetric data and mesh data are needed. Given that the dental volumetric data v. mesh data are presented in different coordinate frames and cannot be used directly, the need arises to find 
a transformation to merge multiple datasets into a globally integrative coordinate frame as a first step for many dental applications. Such a process is often known as registration.

The registration plays a critical role in dental CAD systems, specifically, the accuracy of the registration. In some commercial systems, such as Blue Sky Plan (Blue Sky Bio, LLC, USA), interactive registration methods are used, which require much time and human resources due to excessive demands on purely manual interactions. To date, several automatic registration methods have emerged (Dai et al., 2017; Gao et al., 2007; Mei et al., 2014; Tao \& Da, 2013); however, if a high precision of the registration is needed, the method would be time-consuming. A key solution is to develop a registration method that offers a good trade-off between precision and time cost. Inspired by the low computational complexity of the interactive registration method and the high precision of the automatic registration method, a two-stage registration method is hereby proposed. The proposed method is designed for faster convergence and smaller registration errors.

The rest of this paper is organized as follows. Section 2 overviews the background information focussing on related work, while Section 3 details the various steps to be executed in the novel method being proposed. Section 4 then shifts focus towards examining the experimental analysis results and discussion. Finally, Section 5 closes the paper with insights into some limitations of the proposed method and highlights potential future work.

\section{BACKGROUND}

Notably, dental volumetric data and mesh data are obtained from different scanners, making the multi-modality registration a non-trivial problem. One noteworthy solution is to convert these data to point cloud. In previous work, many point cloud registration methods have been advanced (Dai et al., 2017; Gao et al., 2007; Tao \& Da, 2013; Yuan et al., 2016).

Calling for a high accuracy in the registration, an automatic point cloud registration method based on per-vertex normal (Dai et al., 2017; Gao et al., 2007; Tao \& Da, 2013) was previously proposed. Notwithstanding, such a method is unduly time-consuming because of having to compute the normal. In Xue et al. (2014), feature points were extracted based on the curvature and an implemented coarse registration vis-à-vis an improved three-point translational transformation method. Sadly, in the process of extracting the feature points based on the curvature, the threshold needs to be set manually, which in turn causes grave inconveniences to the user. In Yuan et al. (2016), an improved Iterative Closest Point (ICP) algorithm was proposed. In this method, the principal component analysis (PCA) was used to achieve the initial registration of the point clouds. Then, the k-dimensional tree was used to speed up the search, and a threshold of the angle between the direction vectors was set to remove the wrong point pairs to improve the efficiency of the algorithm.

Unfortunately, for point clouds without conspicuous geometric features, the algorithm cannot improve the accuracy of the registration as the angles between the direction vectors of the point pairs were too small. Jung et al. (2015) manually selected the feature points and then used the ICP algorithm for registration. However, the location accuracy was limited by the experience of the user and the operating state, and the registration method is not accurate. In order to solve this problem, Dai et al. (n.d.) proposed a multi-point alignment method using a thermosetting resin to produce an occlusion splint with several alumina ceramic balls to realize a rapid registration of optical scanning crown data and CT data. In this method, the registration accuracy is high, but the drawback results in an extra burden for the patients. In Yang et al. (2017), a Procrustes analysis based ICP (PICP) method is proposed. The PICP method used the Procrustes analysis to solve the least-squares function of the point cloud data to improve the registration accuracy. Since the amount of data and the components are different, it cannot apply to the registration of volumetric data and mesh data. Even so, while the two-way search method improves accuracy, it still is time-consuming.

Altogether, prior research on registering the dental volumetric data with the dental mesh data has been inadequate. Further, existing registration methods based on point cloud is unsuited for multi- 
modality registration for the following reasons. First, the surface model and the dental mesh are quite different in terms of pose, scale, and data structure, especially inconsistency in scale. Second, the interactive method is rough as the interaction is severely susceptible to user's experience. Finally, the automatic method is time-consuming if the registration accuracy has to be high. Fortunately, the aforementioned problems can be resolved via a two-stage registration method as proposed herein.

The main contributions of this work may thus be summarized as follows:

1. Converting the multi-modality registration problem into a point cloud registration problem, and then proposing a two-stage method to offer a tradeoff between precision and time cost for the registration;

2. Introducing a scale transformation to solve the problem of inconsistency in scale; and,

3. Speeding up the registration by via stridden sampling.

\section{METHOD}

Improving the accuracy of registration and avoiding tedious interactions, the registration method proposed here includes two stages: (a) rough registration with interactions only needed in this stage; and, (b) precise registration. With the help of the precise registration stage, the accuracy of registration is not affected by subtle differences emerging between points that are selected interactively.

Prior to discussing the technical details on our approach, the overall procedure will be presented first. The two-stage registration method for dental volumetric data and mesh data encompasses four (4) key steps as shown in Figure 1: data input, data preprocessing, rough registration, and precise registration. As shown, the overall procedure of the proposed method includes, firstly, dental volumetric data and dental mesh data input.

Following the data input, the region of interest (ROI) is set to delete unwanted or useless parts of the volumetric data. The surface model is reconstructed from the volumetric data; then, the nonmanifold edges, degenerate faces, inconsistent normal, and other components in the dental mesh are repaired. The mesh data is further segmented to keep the information containing the crown. All of these activities entail the data preprocessing, which is further detailed below.

Importantly, two sets of data are constructed by interactively selecting points from the constructed surface mode and the dental mesh data respectively. Based on the point sets, the rough registration is then performed. In the precise registration stage, stridden sampling is used to reduce the computation complexity. Finally, the result can be obtained by the proposed registration algorithm.

Below, the proposed method is elaborated in detail, including data preprocessing (Subsection 3.1), rough registration (Subsection 3.2), stridden sampling (Subsection 3.3), and precise registration with scale transformation (Subsection 3.4).

\subsection{Data Preprocessing}

The volumetric data and dental mesh data being gathered will contain some unwanted or useless data (noises). To eliminate these noises, they are deleted by setting ROI in the volumetric data. For dental mesh, after repairing, a dental segmentation algorithm ( $\mathrm{Li}$ et al., 2007) is used to separate the teeth crown from the other parts, as shown in Figure 2.

As shown in Figure 3, the world coordinates, instead of pixel coordinates, are required in the rough registration stage; hence, the marching cube algorithm (Masala et al., 2013) is applied to the volumetric data to reconstruct the surface model. Moreover, given that the surface model is aligned with the volumetric data, the problem of registering the mesh data with the dental volumetric data is resolved by transforming the mesh data into those consistent with the volumetric data that are aligned with the surface model. 


\section{Figure 1. Overall Procedure}

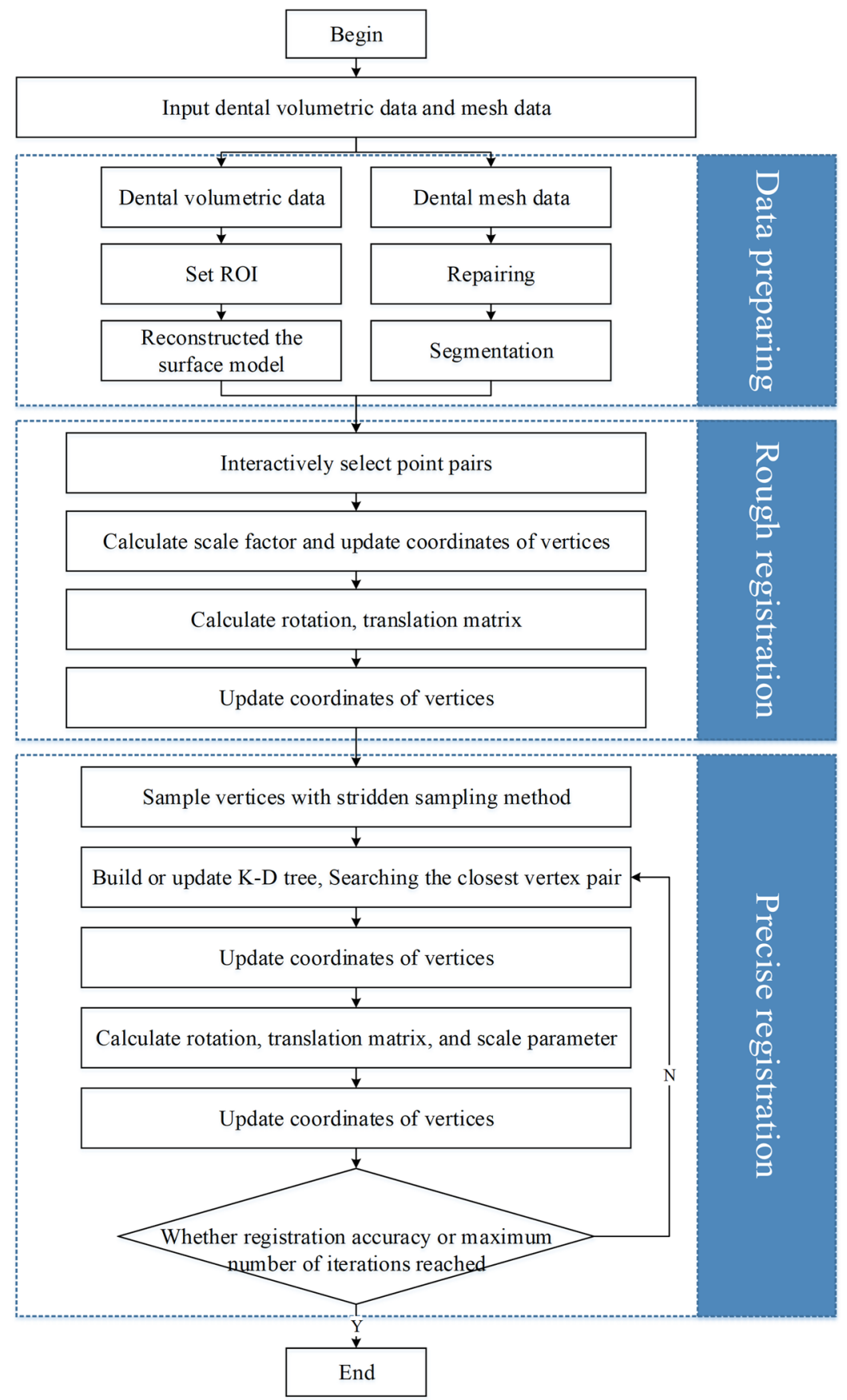



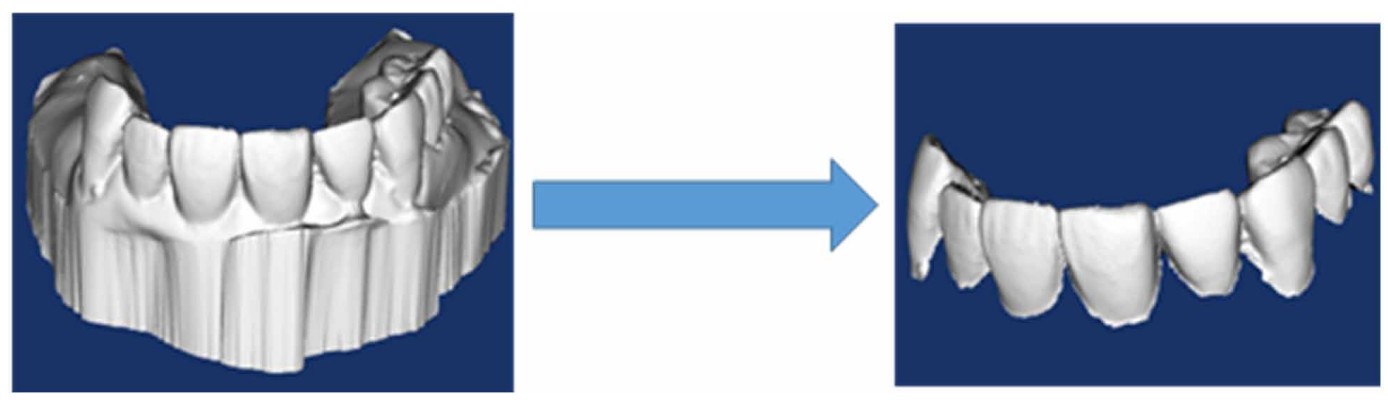

Figure 3. Surface Model
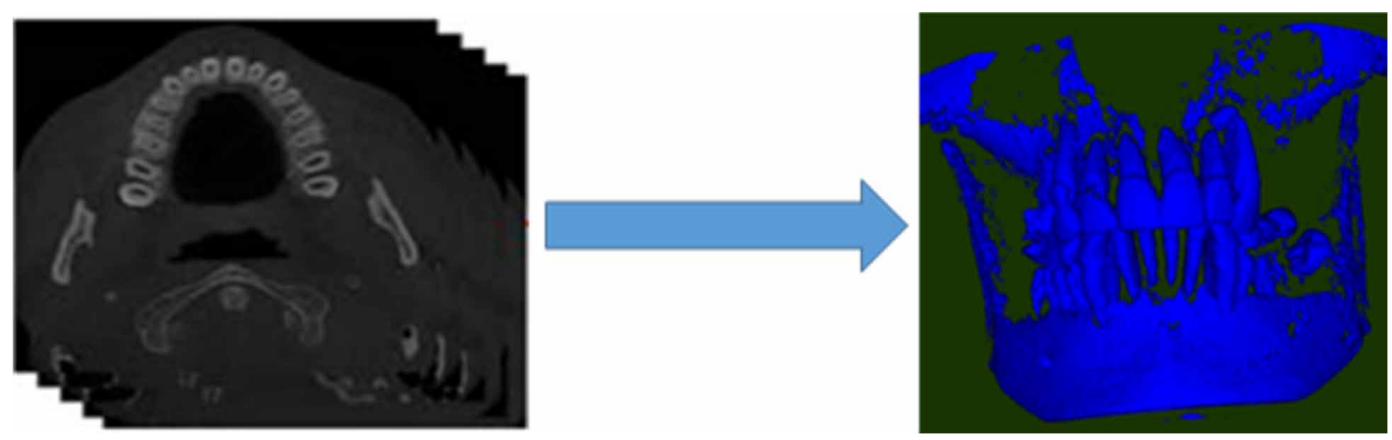

\subsection{Rough Registration}

In the traditional registration, the ICP algorithm proposed by Besl \& Mckey (1992) is commonly used. However, the surface model and the dental mesh are quite different in terms of pose, scale, and the number of vertices. As such, the ICP algorithm will be too costly for rough registration implementation. At this stage, a semi-automatic method based on unit quaternion is used.

Attending to the problem of scale inconsistency, one of the models needs to be scaled. The computation to perform this are as follows:

If $p$ and $u$ are the coordinates of the vertices of the dental mesh and surface mode respectively, the scale factor $s$ is calculated based on the equation (1).

$$
\begin{gathered}
s=\frac{1}{m} \sum_{i=1}^{m} \frac{\left|p_{i+1}-p_{i}\right|}{\left|u_{i+1}-u_{i}\right|} \\
p_{\text {new }(i)}=\frac{p_{i}}{s}
\end{gathered}
$$

Then, $p$ is replaced by $p_{n e w}$.

Notably, the surface model and the dental mesh can be seen as two (2) point sets. The key to the rough registration is to find a suitable transformation, including translation and rotation. As discussed below, the unit quaternion method (Besl \& Mckay, 1992; Dai et al., 2007; Mohamad et al., 2015) is simple, but effective. 
If the target point set $X$ and the reference point set $Y$ is the input of the method, they should satisfy the following constraints:

The point number in $X$ is equal to the point number in $Y$,

$N_{X}=N_{Y}$; each point in $X$ should have its corresponding point in $Y$.

To satisfy the constraints, $m(m \geq 3)$ points are selected interactively from the surface model and the dental mesh respectively to construct the two point set $X$ and $Y$.

If the rotation vector is set as a unit quaternion $y_{R}=\left[y_{0}, y_{1}, y_{2}, y_{3}\right]^{T}$, where $y_{0} \geq 0, y_{0}{ }^{2}+y_{1}{ }^{2}+y_{2}{ }^{2}+y_{3}{ }^{2}$ $=1$, the rotation matrix $R\left(y_{R}\right)$ can be written as:

$$
R\left(y_{R}\right)=\left[\begin{array}{ccc}
y_{0}{ }^{2}+y_{1}{ }^{2}-y_{2}{ }^{2}{ }^{2}{ }_{3}{ }^{2} & 2\left(y_{1} y_{2}-y_{0} y_{3}\right) & 2\left(y_{1} y_{3}+y_{0} y_{2}\right) \\
2\left(y_{1} y_{2}+y_{0} y_{3}\right) & y_{0}{ }^{2}-y_{1}{ }^{2}+y_{2}{ }^{2}-y_{3}{ }^{2} & 2\left(y_{2} y_{3}-y_{0} y_{1}\right) \\
2\left(y_{1} y_{3}-y_{0} y_{2}\right) & 2\left(y_{2} y_{3}+y_{0} y_{1}\right) & y_{0}{ }^{2}-y_{1}{ }^{2}-y_{2}{ }^{2}+y_{3}{ }^{2}
\end{array}\right] .
$$

If the translation vector is set as $y_{T}=\left[y_{4}, y_{5}, y_{6}\right]^{T}$, the transformation vector is $y=\left[y_{R} \mid y_{T}\right]^{T}$. The rotation and translation can be obtained by solving the optimization problem as:

$\min _{y_{R}, y_{T}} \frac{1}{N_{x}} \sum_{i=1}^{N_{x}}\left\|y_{i}-R\left(y_{R}\right)_{x_{i}}-y_{T}\right\|^{2}$.

The solutions of the equation (3) can be obtained by the method as follows:

Calculating the mean and covariance.

$\mu_{x}=\frac{1}{N_{x}} \sum_{i=1}^{N_{x}} x_{i}$

$\mu_{y}=\frac{1}{N_{y}} \sum_{i=1}^{N_{y}} y_{i}$

$$
U=\operatorname{cov}(X, Y)=\frac{1}{N_{y}} \sum_{i=1}^{N_{y}}\left(x_{i}-\mu_{x}\right)\left(y_{i}-\mu_{y}\right)^{T}
$$

Constructing a $4 \times 4$ symmetric matrix $T$ from the covariance matrix $U$, 
$T=\left[\begin{array}{cc}\operatorname{tr}(U) & \Delta^{T} \\ \Delta & U+U^{T}-\operatorname{tr}(U)_{I_{3}}\end{array}\right]$,

where $I_{3}$ is a $3 \times 3$ identity matrix, $\operatorname{tr}(U)$ is the trace of $U$, and $\Delta=\left[A_{23}, A_{31}, A_{12}\right]^{T}, A_{i j}=\left(R-R^{T}\right)_{i, j}$.

(3) Calculating the eigenvalue and eigenvector of the matrix $T$, and the eigenvector corresponding to the largest eigenvalue is the rotation vector $y_{R}$, and the translation vector is $y_{T}=\mu_{y}-R\left(y_{R}\right) \mu_{x}$.

\subsection{Stridden Sampling}

The dental mesh and the surface model are constructed by triangles, which comprise three vertices. During registration, only the coordinates of these vertices are used, realizing that computational complexity is positively correlated with the number of vertices.

In order to reduce the computational complexity, some vertices in the region without obvious features should be ignored. Fortunately, vertices of the dental mesh and the surface model are dense in the region with obvious features, while being sparse in the flat region. Hence, the stridden sampling method is used.

If there are $\mathrm{N}$ vertices in the dental mesh and $\mathrm{n}$ vertices are needed, vertices with the index $1, \mathrm{k}+1$, $2 k+1, \ldots, n * k+1(k=[N / n])$ are sampled. Figure 4 depicts the sampling result, where the number of vertices is $30361, \mathrm{n}=1031, \mathrm{k}=30$, and the red dot is the sampled vertices. It is obvious that the number of sampled vertices in the red circle is more than ones in the blue circle, and the region of the blue circle is flatter than that of the red circle.

\section{Figure 4. Vertices Sampling}

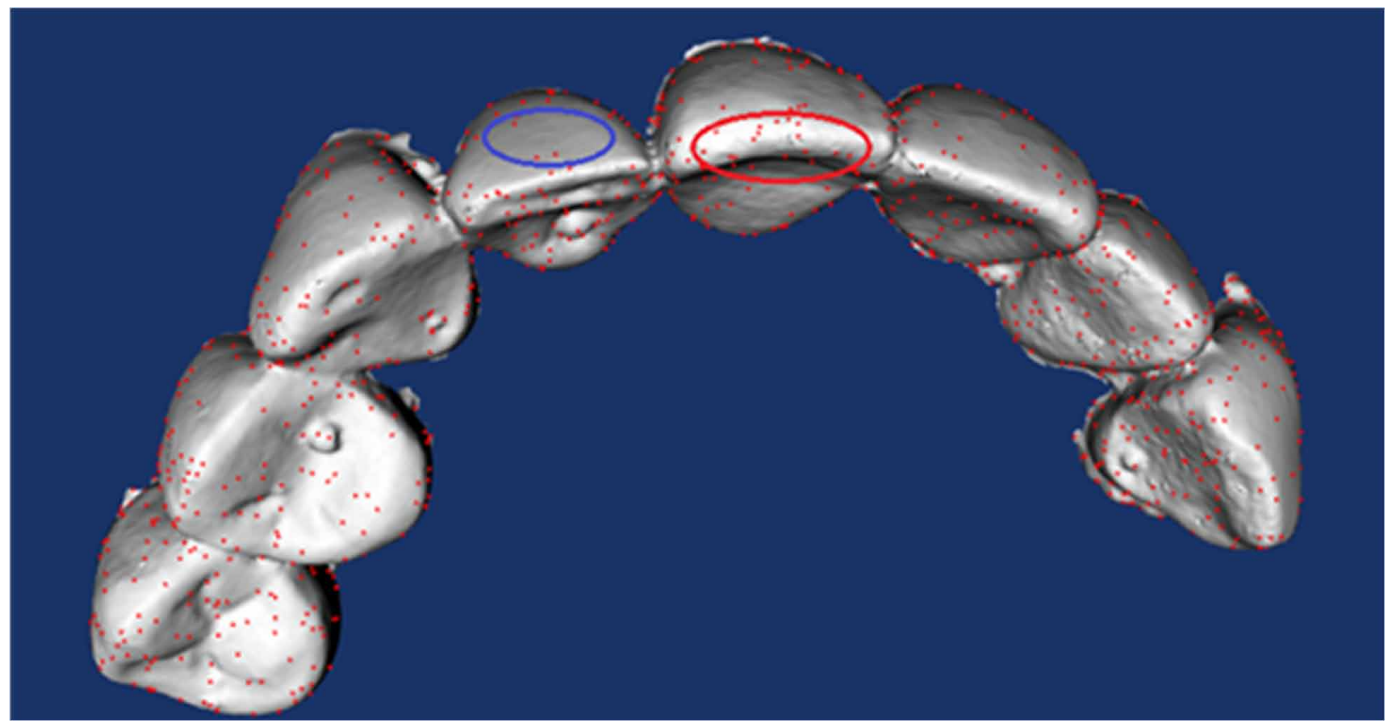

In Figure 4, it is apparent that the number of sampled vertices in the red circle is more than ones in the blue circle, and the region of the blue circle is flatter than the red circle. The proposed method is simple but efficient. As compared with the random sampling method, stridden sampling 
can sample representative points. Also, when compared with the k-means center sampling method, it is more time saving.

\subsection{Precise Registration with Scale Transformation}

After the rough registration, the dental mesh and the surface model are roughly aligned. Nonetheless, in the rough registration stage, there may be errors caused by interactions. Accordingly, automatic precise registration is one way to solve the problem. Based on the traditional ICP algorithm, an improved ICP method with scale transformation is proposed, as shown in Algorithm 1.

The key to solving the optimization problem would be to use:

$$
\min _{R, T} \sum_{i=1}^{n}\left\|L_{i}-\left(\lambda R H_{i}+T\right)\right\|^{2}
$$

where $\mathrm{Hi}$ is the coordinates of the vertex in the surface model, $\mathrm{Li}$ is the coordinates of the vertex in the dental mesh, which is closest to $\mathrm{Hi}, \mathrm{R}$ and $\mathrm{T}$ are rotation matrix and translation matrix respectively, and 1 is the scale parameter, which can be computed via the equation:

$$
\lambda=\sqrt{\frac{\sum_{i=1}^{n}\left\|L_{i}\right\|^{2}}{\sum_{i=1}^{n}\left\|H_{i}\right\|^{2}}}
$$

In the precise registration stage, the closest vertex searching is frequently used. To improve efficiency, the K-D tree (Liu et al., 2016) is recommended. The K-D tree, which is proved to reduce the time cost by ICP (Greenspan \& Yurick, 2003; Li et al., 2017; Nchter et al., 2007), is a binary searching tree for which each node represents a partition of the k-dimensional space. The time complexity of searching the nearest point via the K-D tree is $\mathrm{O}(\mathrm{nlogn})$, which can significantly improve the nearest point search speed in the point cloud.

Registration error e is an essential parameter in the iteration of the method, which is defined as

$$
\varepsilon=\sqrt{\frac{\sum_{i=1}^{n}\left|L_{i}-H_{i}\right|^{2}}{n}} .
$$

\section{RESULTS AND DISCUSSION}

In this section, the performance of the proposed method is evaluated. All software programs, compiled in Microsoft Visual Studio 2012 and run on a computer with 2 GB RAM, Intel ${ }^{\circledR}$ Core $^{\mathrm{TM}}$ i5-3470 3.2 GHz CPU, and Windows 764 bits system, have been implemented in C++ depending on VTK (The Visualization Toolkit) 7.0.0 (Schroeder et al., 2004).

The registration results via the proposed method are shown in Figure 5 The first column shown is the surface model reconstructed from the volumetric (CT) data. The second column entails the dental mesh data being segmented from the optical surface scan data, which are acquired by traditional 
Algorithm 1. Precise Registration

\begin{tabular}{|c|c|c|}
\hline Input: & & The dental mesh $L$ and the surface model $H$ \\
\hline \multicolumn{2}{|l|}{ Output: } & $\begin{array}{c}\text { Rotation matrix } \mathbf{R} \text {, translation matrix } \mathbf{T} \text {, and scale parameter } \\
\lambda\end{array}$ \\
\hline & -1 & $\begin{array}{l}\text { Sampling } \mathrm{n} \text { vertices from } \mathrm{L} \text { and } \mathrm{H} \text { using the method in subsection } \\
3.3 \text { respectively; }\end{array}$ \\
\hline & -2 & Constructing or updating the K-D tree; \\
\hline & -3 & Searching the closest vertex pair $\mathrm{H}_{\mathrm{i}}$ and $\mathrm{L}_{\mathrm{i}}$ based on K-D tree; \\
\hline & -4 & Calculating $\lambda$ based on equation (9); \\
\hline & -5 & Solving $\mathrm{R}$ and $\mathrm{T}$ based on the method in subsection 3.2 ; \\
\hline & -6 & Updating the coordinates of the vertices in $\mathrm{L}$ and $\mathrm{H}$; \\
\hline & -7 & $\begin{array}{l}\text { Calculating the registration error } \varepsilon \text { based on equation (10); if } \varepsilon<\mathrm{t}(\mathrm{t} \\
\text { is a threshold) or the maximum number of iterations reached, the } \\
\text { algorithm ends; otherwise, repeat step (2). }\end{array}$ \\
\hline
\end{tabular}

Figure 5. Registration Results

Case A
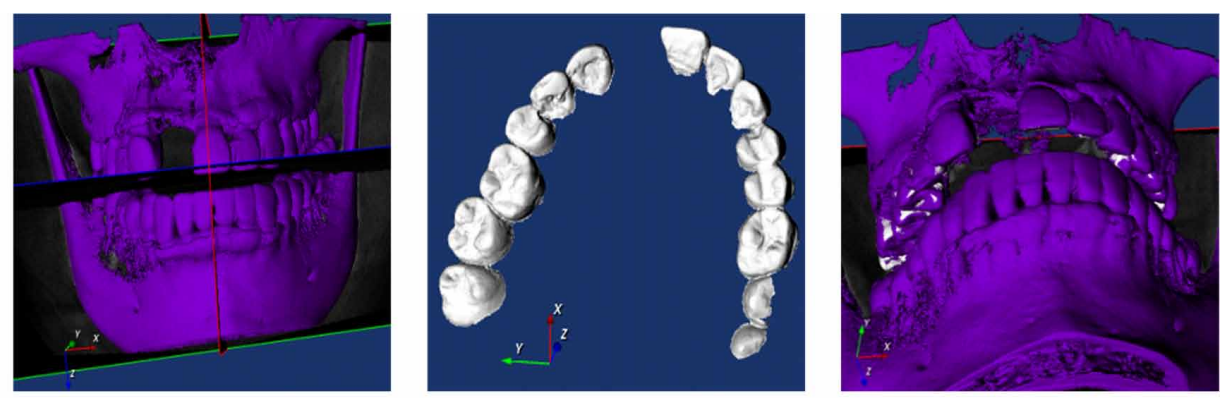

Case B
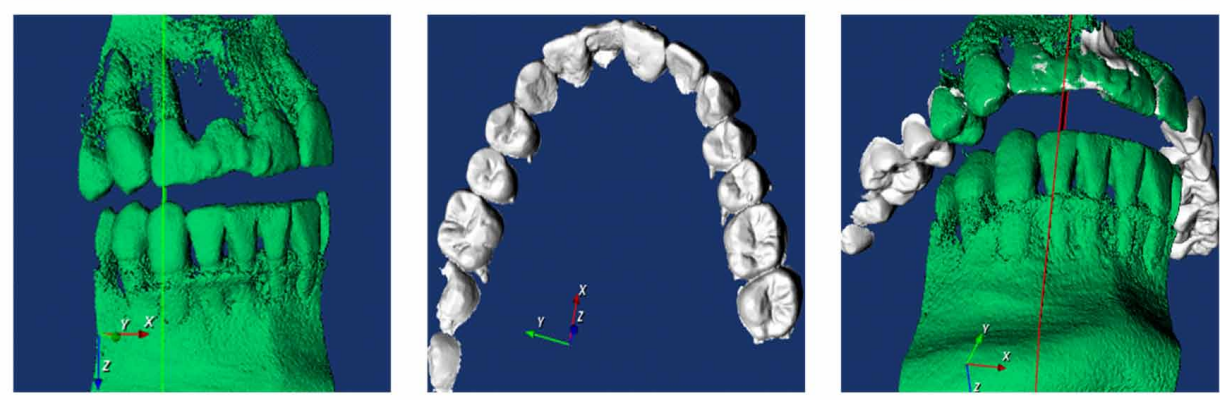

Case C
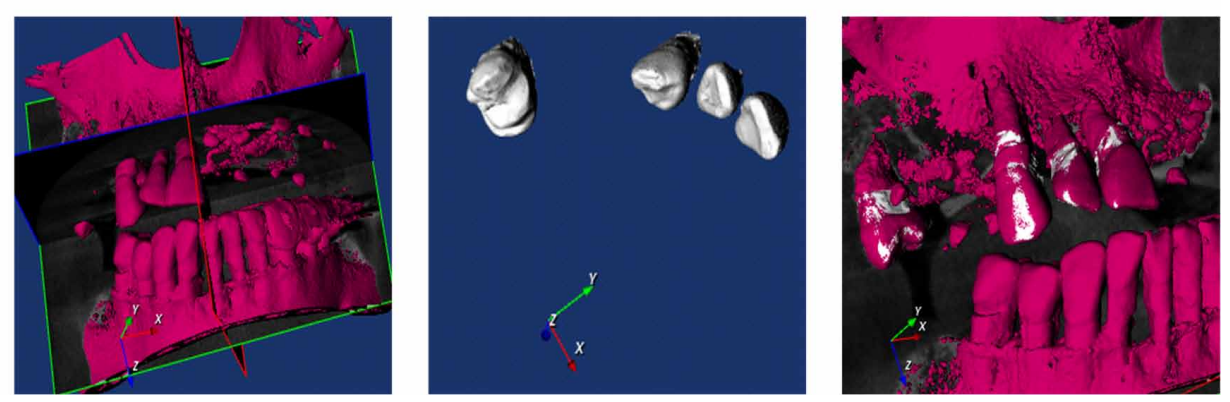
impression followed by 3D scanning. The third column depicts the registration results. Case A evaluates the proposed method on the maxillary data with missing a tooth. In Case B, as a fallout from the missing part of the volumetric data, the reconstructed surface model only contains several teeth in the front. In Case $\mathrm{C}$, only four maxillary teeth in the segmented dental mesh are present. The registration results from the third column demonstrate that the proposed method is effective and accurate for the missing teeth even in the face of incomplete data.

In our experiment, the proposed method is compared vis-à-vis the traditional ICP (Besl \& Mckay, 1992) and PCA+ICP (Yuan et al., 2016). Figure 6(a) shows the reconstructed surface model and the teeth mesh segmented from the corresponding dental mesh. More specifically, Figure 6(b) unveils the fact that the traditional ICP fails to register the two datasets. Figures 6(c-d) detail the registration

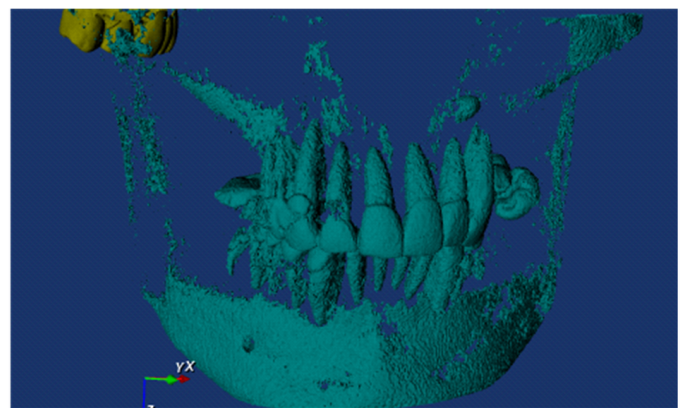

(a) The reconstructed surface model.

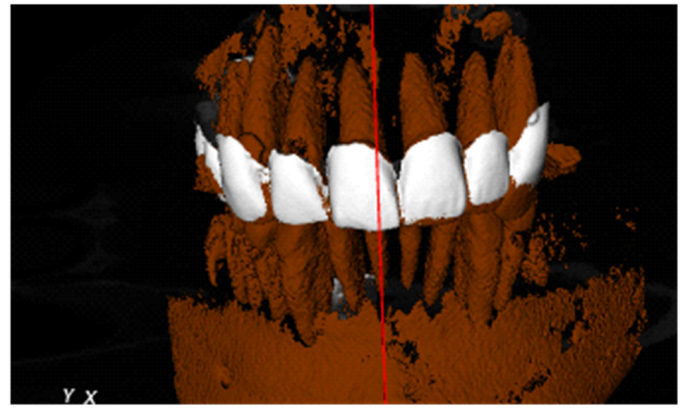

(c) The registration result with $\mathrm{PCA}+\mathrm{ICP}$

[9] in viewport 1.

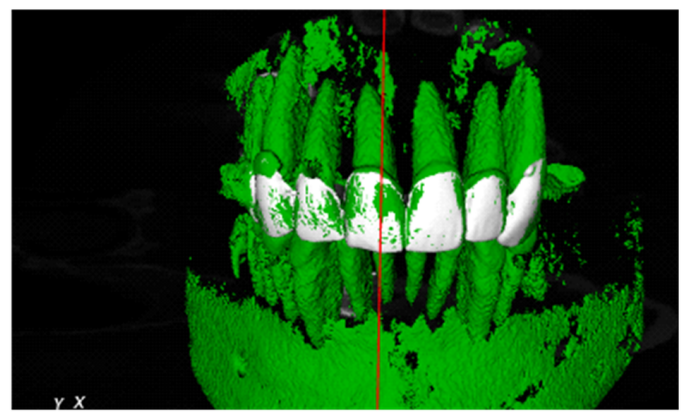

(e) The registration result with our method in viewport 1 .

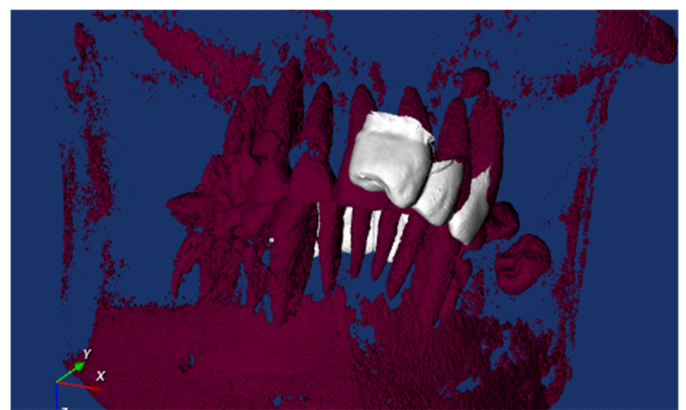

(b) The registration result with ICP [14]

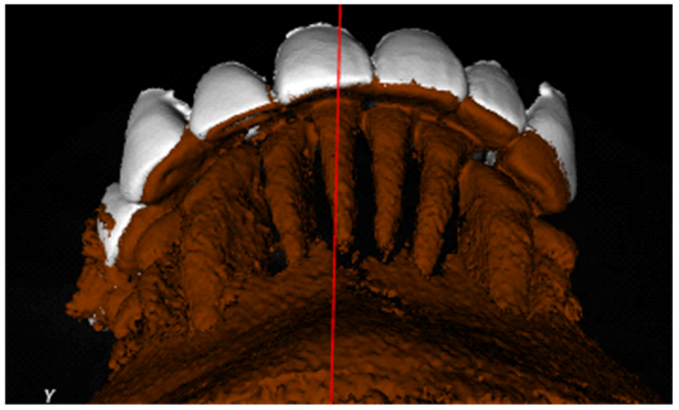

(d) The registration result with $\mathrm{PCA}+\mathrm{ICP}$

[9] in viewport 2

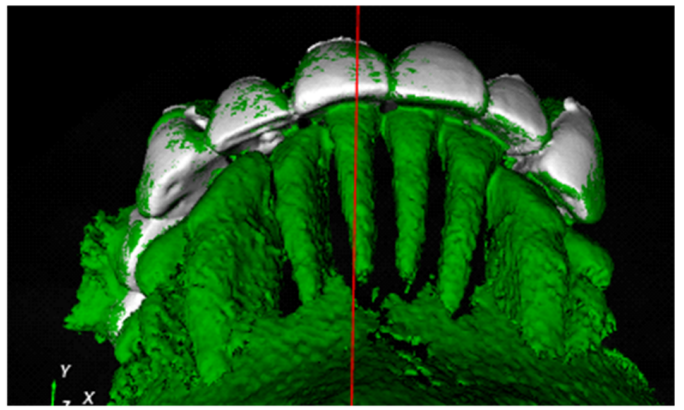

(f) The registration result with our method in viewport 2 . 
results of PCA+ICP, whereas Figures 6(e-f) present the results of the proposed method. Apparently, the dental mesh (i.e., white part) completely wraps the surface model (i.e., red part) in Figures 6(cd). Conversely, in Figures 6(e-f), the dental mesh (white part) is fused with the surface model (i.e., green part). Put simply, the experimental results show that the proposed method yields more accurate registration results than via ICP and PCA+ICP.

In order to validate that scale transformation and stridden sampling are effective for registration, the traditional method without scale transformation, the traditional method without stridden sampling, and the proposed method are evaluated respectively. Results are reported in Figure 7(a-c). As shown in Figure 7(a), the reconstructed surface model cannot align well with the dental mesh because the scale of the volumetric data is different from that of the dental mesh. Comparing Figure 7(b) with Figure 7(c), we find that the results are similar, but that in speeding up the registration, the stridden sampling significantly reduces the size of the point set. Hence, the implementation of the scale
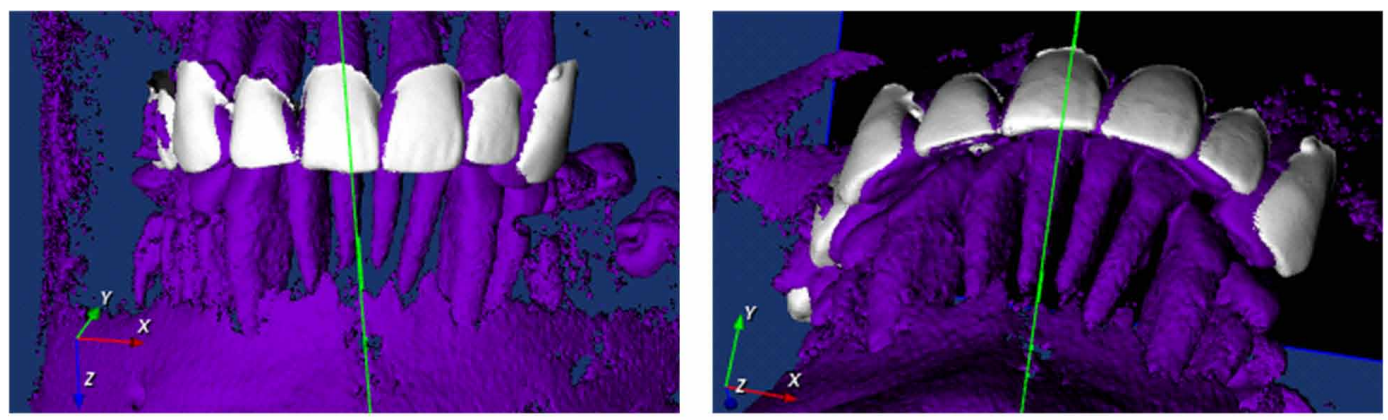

(a) Without scale transformation.
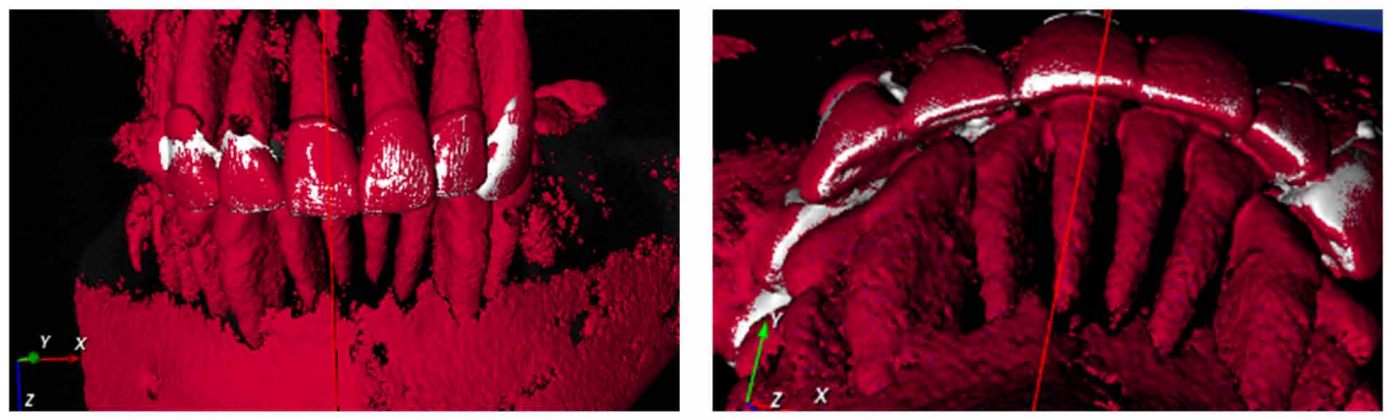

(b) Without stridden sampling.
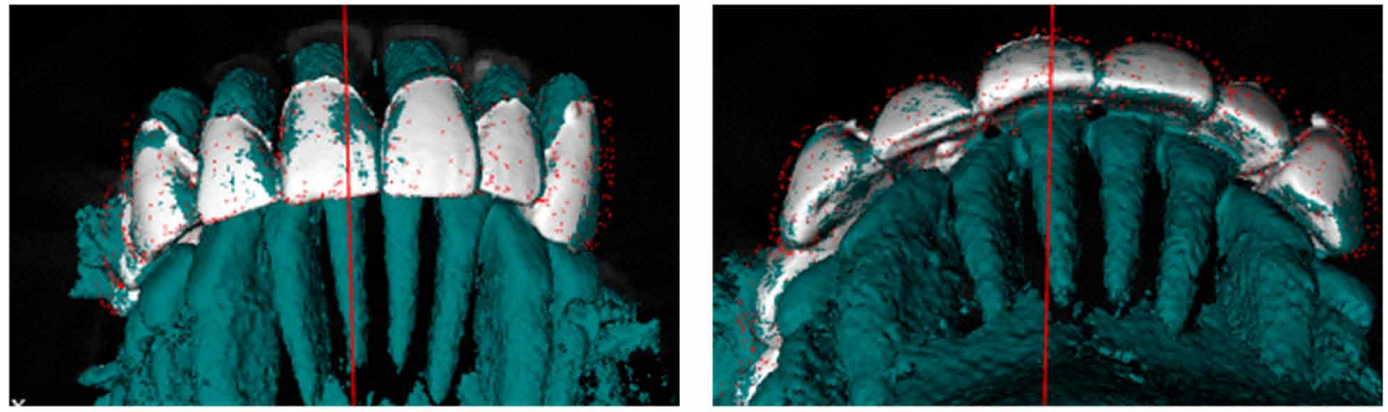

(c) With scale transformation and stridden sampling. 
transformation solves the problem of scale inconsistency and improves the accuracy. As well, the stridden sampling enhances the registration speed.

Table 1The proposed two-stage registration method offers a good trade-off between precision and time cost with the help of interactively selected point sets and precise registration stage.

A quantitative experiment is performed with ten (10) cases to assess the precision and time cost of registration. Here, the average time cost and standard deviation are also recorded with the results shown in Table 1[REMOVED REF FIELD], where the precision is the threshold $t$ as described in Algorithm 1 step (7).

Table 1. The precision and time cost of different registration methods

\begin{tabular}{|c|c|c|c|}
\hline Method & Precision (threshold $t$ ) & Average Time Cost (s) & Standard Deviation \\
\hline ICP (Besl \& Mckay, 1992) & 0.1 & 2.680 & 0.142 \\
\hline PCA+ICP (Yuan et al., 2016) & 0.1 & 3.152 & 0.170 \\
\hline Our method & 0.1 & 2.554 & 0.109 \\
\hline Our method & 0.001 & 2.858 & 0.147 \\
\hline Our method & 0.0001 & 2.853 & 0.122 \\
\hline $\begin{array}{c}\text { Our method without stridden } \\
\text { sampling }\end{array}$ & 0.1 & 3.243 & 0.149 \\
\hline
\end{tabular}

According to Table 1, the time cost of the proposed method gradually increases with graduated improved precision. However, the growth of time cost is significantly lower than that of precision, which means that high-precision registration results can be obtained almost in real-time. Comparing the third row and the last row of Table 1, we see evidence of the stridden sampling speeding up the registration process. Examining the first three rows of Table 1, the time cost of the proposed method is least, although the time cost of ICP is almost the same as that of the proposed method; moreover, the registration result of ICP is incorrect, as shown in Figure 6(b).

As shown in Figure 8, given the same number of iterations, the registration error of the proposed method is noted to be the smallest, with the least time cost but with the same registration accuracy.

As depicted in Figure 1 and Figure 5, one limitation of the proposed method is the need to reconstruct the volumetric data when using the proposed method. 


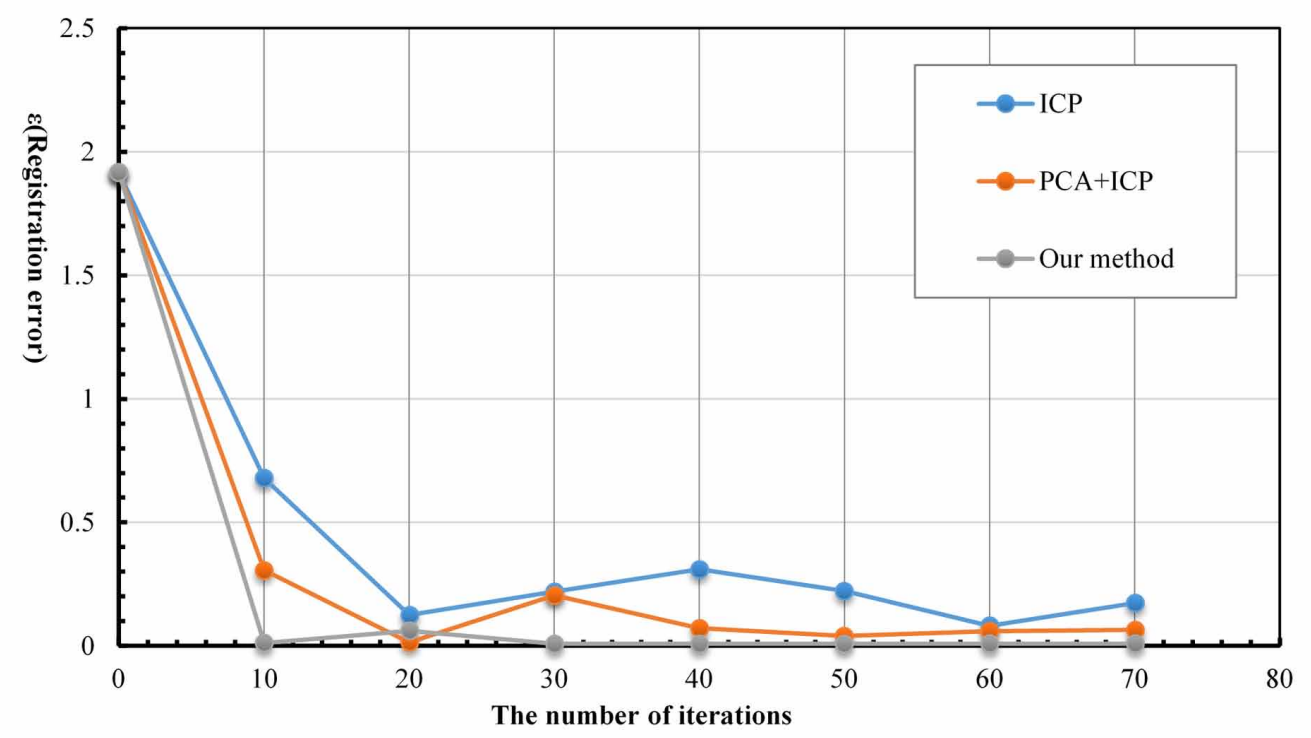

\section{CONCLUSION}

In summary, this work contributes to our understanding of how the registry of the dental volumetric data with the dental mesh can be enhanced via a two-stage registration method. The method introduces scale transformation to solve the problem of scale inconsistency, and stridden sampling to enhance the registration speed. Moreover, our study results show that the two-stage registration method offers a good trade-off between precision and time cost.

In term of study limitations, this work is limited in terms of the need to reconstruct the volumetric data. The challenge may be overcome by directly sampling points from the volumetric data.

For potential future work, an overarching aim is not only to improve the stability and performance of the proposed method, but also to implement registration without volumetric data reconstruction. Moreover, it will be an interesting work to extend the method to other fields such as Lidar data from the self-driving car.

\section{Conflicts of Interest}

The authors declare that there are no conflicts of interest regarding the publication of this paper. 


\section{REFERENCES}

Besl, P. J., \& Mckay, H. D. (1992). A method for registration of 3-D shapes. Pattern Analysis and Machine Intelligence. IEEE Transactions on Pattern Analysis and Machine Intelligence, 14(2), 239-256. doi: $10.1109 / 34.121791$

Dai, Li, \& Yang. (n.d.). Research and primary evaluation of an automatic fusion method for multisource tooth crown data. Int J Numer Meth Biomed Engng.

Dai, J. L., Chen, Z. Y., \& Ye, X. Z. (2007). The Application of ICP Algorithm in Point Cloud Alignment. Journal of Image and Graphics, 12(3), 517-521.

Dai, J. L., Chen, Z. Y., \& Ye, X. Z. (2017). The application of ICP algorithm in point cloud alignment. Journal of Image and Graphics, 12(3), 517-521.

Gao, H., \& Chae, O. (2010). Individual tooth segmentation from CT images using level set method with shape and intensity prior. Pattern Recognition, 43(7), 2406-2417. doi:10.1016/j.patcog.2010.01.010

Gao, P. D., Peng, X., \& Li, A. M. (2007). Depth image registration with ICP frame using surface mean interspace measure. Journal of Computer-Aided Design \& Computer Graphics, 19(6), 719-724.

Greenspan, M., \& Yurick, M. (2003). Approximate K-D Tree Search for Efficient ICP. Fourth International Conference on 3-D Digital Imaging and Modeling, 442. doi:10.1109/IM.2003.1240280

Hämmerle, Cordaro, Van Assche, Benic, Bornstein, Gamper, \& Gotfredsen. (2015). Digital technologies to support planning, treatment, and fabrication processes and outcome assessments in implant dentistry. Clinical Oral Implants Research, 26, 97-101.

Jung, W., Park, S., \& Shin, H. (2015). Combining volumetric dental CT and optical scan data for teeth modeling. Computer Aided Design, 67, 24-37. doi:10.1016/j.cad.2015.04.008

Li, S. H., Liang, Z. Q., \& Lin, S. (2017). Estimating clumping index of woody canopy with terrestrial lidar data. Geoscience and Remote Sensing Symposium (IGARSS), 5790-5793. doi:10.1109/IGARSS.2017.8128324

Li, Z. L., Ning, X. J., \& Wang, Z. B. (2007). A Fast Segmentation Method for STL Teeth Model. 2007 IEEE/ ICME International Conference on Complex Medical Engineering, 163-166. doi:10.1109/ICCME.2007.4381713

Liu, J., Zhu, J. W., Yang, J. L., \& Meng, X. L. (2016). Three-dimensional point cloud registration based on ICP algorithm employing K-D tree optimization. Eighth International Conference on Digital Image Processing (ICDIP 2016).

Masala, G. L., Golosio, B., \& Oliva, P. (2013). An improved Marching Cube algorithm for 3D data segmentation. Computer Physics Communications, 184(3), 777-782. doi:10.1016/j.cpc.2012.09.030

Mei, X., Li, Z., Xu, S., \& Guo, X. (2014). Registration of the Cone Beam CT and blue-ray scanned dental model based on the improved ICP algorithm. International Journal of Biomedical Imaging, 2014, 1-8. doi:10.1155/2014/348740 PMID:24511309

Mohamad, M., Ahmed, M. T., Rappaport, D., \& Greenspan, M. A. (2015). Super Generalized 4PCS for 3D Registration. 2015 International Conference on $3 D$ Vision (3DV).

Nchter, Kai, \& Hertzberg. (2007). Cached k-d tree search for ICP algorithms. IEEE International Conference on 3-D Digital Imaging \& Modeling, 419-426.

Pauwels, R., Stamatakis, H., Bosmans, H., Bogaerts, R., Jacobs, R., Horner, K., \& Tsiklakis, K. (2013). Quantification of metal artifacts on cone beam computed tomography images. Clinical Oral Implants Research, 24, 94-99. doi:10.1111/j.1600-0501.2011.02382.x PMID:22168574

Schroeder, W. J., Lorensen, B., \& Martin, K. (2004). The visualization toolkit: an object-oriented approach to $3 D$ graphics. Kitware.

Tao, H. J., \& Da, F. P. (2013). Automatic registration algorithm for the point cloud based on the normal vector. Chinese Journal of Lasers, 2013(8), 179-184. 
Van Noort, R. (2012). The future of dental devices is digital. Dental Materials, 28(1), 3-12. doi:10.1016/j. dental.2011.10.014 PMID:22119539

Wu, T., \& Zhang, L. B. (2016). Three dimension tooth reconstruction using level set active contour model. Journal of Image and Graphics, 21(8), 1078-1087.

Yang, L., Qiao, Z. S., Chen, L. L., \& Yang, Z. P. (2017). PICP Registration Method Based on Procrustes Analysis and ICP Algorithm. Journal of Computer-Aided Design \& Computer Graphics, 29(2), 337-343.

Yuan, Yu, \& Luo. (2016). 3D point cloud matching based on principal component analysis and iterative closest point algorithm. 5th International Conference on Audio, Language and Image Processing, 404-408.

Zhang, D. X., Gan, Y. Z., \& Xiong, J. (2017). 'Three-dimensional tooth model reconstruction based on fusion of dental computed tomography images and laser-scanned images. Journal of Biomedical Engineering, 34(1), 2-14.

Zhao, Liu, Sun, \& Wang. (2017). Three-dimensional data fusion method for tooth crown and root based on curvature continuity algorithm. Journal of Peking University (Health Sciences), 49(4), 719-723.

Shuai Yang obtained his BE and ME of computer application at Tianjin Normal University in China in 2012 and 2014, respectively. From 2014 to the present, he worked in School of Computer Science and Technology, Tiangong University, China. His research interests include digital geometry processing and image processing.

Wenjing Shi obtained her BE of The Internet of Things at Tianjin Agricultural College in China in 2012, and she is a postgraduate of computer science at school of computer science and technology, Tiangong University in China now, on the research subjects of medical image processing and information visualization.

Yongzhen Ke obtained his BE of computer application at Tianjin Polytechnic University in China in 1997 and received his ME and PhD of computer application at Tianjin University in China in 2000 and 2008, respectively, on the research subjects of image processing and data visualization. From 1997 to the present, he worked in Tiangong University, China. Now he is a professor at School of Computer Science \& Technology, Tiangong University, China. His current research interests include image processing, digital image forensic, intelligent image Analysis, and information visualization.

Yongjiang Xue obtained his ME of Pattern Recognition \& Intelligent Systems at Hebei University of Technology in China in 2009, on the research subjects of robot and computer vision. From 2012 to the present, he worked in School of Computer Science and Technology, Tiangong University, China. 